\title{
Fungal allergen propensity study confirmed through MAST- CLA in southeastern region of KOREA
}

Joonsoo Park, M.D., Ph.D., YongWoo Choi, M.D.

Department of Dermatology, School of Medicine, Catholic University of Daegu, Daegu, Republic of Korea

\section{Background}

Exposure and sensitization to fungal allergens can promote the development and worsening of allergic disease, such as allergic rhinitis, pharyngitis, asthma, airborne dermatitis, or allergic conjunctivitis. The main components of bioaerosol are fungi and their metabolites, which are common in the environment. Allergic patients showing a simultaneously positive reaction to multiple allergens are frequently found.

\section{Objective}

Allergens are the subset of antigens that stimulate an IgEmedicated response. A great number of laboratories are using qualitative or semi-quantitative analyzers as a screening test for allergen, such as MAST CLA (multiple allergen simultaneous test chemilucent assay). But, allergic patients showing a simultaneously positive reaction to multiple allergens are frequently found. The purpose of this study was to analyze the relationship between fungal allergens that mostly found in South Korea and other simultaneously positive results to the allergens of MAST CLA.

\section{Method}

We enrolled 1040 (588 men, 452 women) allergic disease patients examined with MAST CLA positive to 3 different fungus (Alternaria alternata, Aspergillus fumigatus or Cladosporium herbarum) at Daegu Catholic University Medical Center from January 2010 to July 2017.

\section{Result}

Epidemiologic results show men have more positive in fungal allergen than women. Seasonal deviation shows more in summer, but there are not much differences. Otorhinolaryngology was the most common and followed by dermatology. Allergic rhinitis was the most common, urticaria was the second most common, and atopic dermatitis was the third.

\begin{tabular}{|c|c|}
\hline $\begin{array}{l}\text { Variable } \\
(n-1040)\end{array}$ & Variable : Number (\%) \\
\hline Sex & Male : 588 (56.3), Female :452 (43.7) \\
\hline Age & 31.1 years \\
\hline Season & $\begin{array}{l}\text { Spring : } 229 \text { (22.0), Summer : } 311(29.9) \text {, } \\
\text { Autumn : } 233 \text { (22.4), Winter : } 267 \text { (25.7) }\end{array}$ \\
\hline MAST type & Food : 284 (27.3), Inhalant : 756 (72.7) \\
\hline Department & $\begin{array}{c}\text { Otorhinolaryngology : } 423(40.7) \\
\text { Dermatology : } 374(36.0) \\
\text { Internal medicine }: 209(20.1) \\
\text { Pediatric : } 20(1.9) \\
\text { Others : } 14(1.4)\end{array}$ \\
\hline Diagnosis & $\begin{array}{c}\text { Allergic rhinitis }: 436(41.9) \\
\text { Urticaria }: 176(16.9) \\
\text { Atopic dermatitis }: 133(12.8) \\
\text { Allergic contact dermatitis }: 50(4.8) \\
\text { Asthma }: 36(3.5) \\
\text { Drug eruption }: 15(1.4) \\
\text { Eosinophilia }: 6(0.6) \\
\text { Others }: 188(18.1) \\
\end{array}$ \\
\hline
\end{tabular}

Table 1. Demographic and diagnosis variables
In fungal species Alternaria alternata was the most common, followed by Cladosporium herbarum and Aspergillus fumigatus. Patients who have positive results to all of fungal allergens shows average 4.97 different other antigens, who have positive results to two fungal allergens and only one allergen shows average 3.41 and 2.82 each. Statically significant results were obtained when the positive results to all of fungal allergens were compared with the other results (correlation coefficient of spearman was positive correlation coefficient [0.129]).

\section{Conclusion}

It is concluded that epidemiologic results show no significant differences. But the more positive results to fungal allergens of MAST CLA takes, the more positive results to other allergens of MAST CLA gain. So, we should give patient a message of having more positive allergen of fungus, it could be positive for other allergens.

\begin{tabular}{c|c|c|c|c}
\hline Rank & $\begin{array}{c}\text { Cladosporium } \\
\text { herbarum }\end{array}$ & $\begin{array}{c}\text { Aspergillus } \\
\text { fumigatus }\end{array}$ & $\begin{array}{c}\text { Alternaria } \\
\text { alternata }\end{array}$ & $\begin{array}{c}\text { All } \\
\text { simultaneously } \\
\text { positive }\end{array}$ \\
\hline $1^{\text {st }}$ & $\begin{array}{c}\text { Mite pterony } \\
(29.2 \%)\end{array}$ & $\begin{array}{c}\text { Mite pterony } \\
(42.3 \%)\end{array}$ & $\begin{array}{c}\text { Mite pterony } \\
(36.7 \%)\end{array}$ & $\begin{array}{c}\text { Mite pterony } \\
(49.6 \%)\end{array}$ \\
\hline $2^{\text {nd }}$ & $\begin{array}{c}\text { Mite farinae } \\
(27.1 \%)\end{array}$ & $\begin{array}{c}\text { Mite farinae } \\
(40.8 \%)\end{array}$ & $\begin{array}{c}\text { Mite farinae } \\
(33.1 \%)\end{array}$ & $\begin{array}{c}\text { Mite farinae } \\
(41.2 \%)\end{array}$ \\
\hline $3^{\text {rd }}$ & $\begin{array}{c}\text { House dust } \\
(13.2 \%)\end{array}$ & $\begin{array}{c}\text { Rye } \\
(34.7 \%)\end{array}$ & $\begin{array}{c}\text { House dust } \\
(14.3 \%)\end{array}$ & $\begin{array}{c}\text { Cedar } \\
(33.6 \%)\end{array}$ \\
\hline $4^{\text {th }}$ & $\begin{array}{c}\text { Penicillium } \\
(12.5 \%)\end{array}$ & $\begin{array}{c}\text { House dust } \\
(28.6 \%)\end{array}$ & $\begin{array}{c}\text { Cat } \\
(13.1 \%)\end{array}$ & $\begin{array}{c}\text { Penicillium } \\
(32.8 \%)\end{array}$ \\
\hline $5^{\text {th }}$ & $\begin{array}{c}\text { Acarus siro } \\
(9.7 \%)\end{array}$ & $\begin{array}{c}\text { Orch } \\
(28.6 \%)\end{array}$ & $\begin{array}{c}\text { Dog } \\
(10.1 \%)\end{array}$ & $\begin{array}{c}\text { House dust } \\
(27.7 \%)\end{array}$ \\
\hline $6^{\text {th }}$ & $\begin{array}{c}\text { Cat } \\
(8.3 \%)\end{array}$ & $\begin{array}{c}\text { Sweet vernal } \\
\text { grass } \\
(26.5 \%)\end{array}$ & $\begin{array}{c}\text { Alder } \\
(8.0 \%)\end{array}$ & $\begin{array}{c}\text { Acarus siro } \\
(21.0 \%)\end{array}$ \\
\hline $7^{\text {th }}$ & $\begin{array}{c}\text { Rye } \\
(7.6 \%)\end{array}$ & $\begin{array}{c}\text { Dandelion } \\
(24.5 \%)\end{array}$ & $\begin{array}{c}\text { Rye } \\
(7.6 \%)\end{array}$ & $\begin{array}{c}\text { Rye } \\
(16.8 \%)\end{array}$ \\
\hline $8^{\text {th }}$ & $\begin{array}{c}\text { Dog } \\
(6.3 \%)\end{array}$ & $\begin{array}{c}\text { Reed } \\
(24.5 \%)\end{array}$ & $\begin{array}{c}\text { Acarus siro } \\
(7.4 \%)\end{array}$ & $\begin{array}{c}\text { Candida } \\
(16.8 \%)\end{array}$ \\
\hline
\end{tabular}

Table 2. Simultaneously positive allergens observed in each fungal allergen.

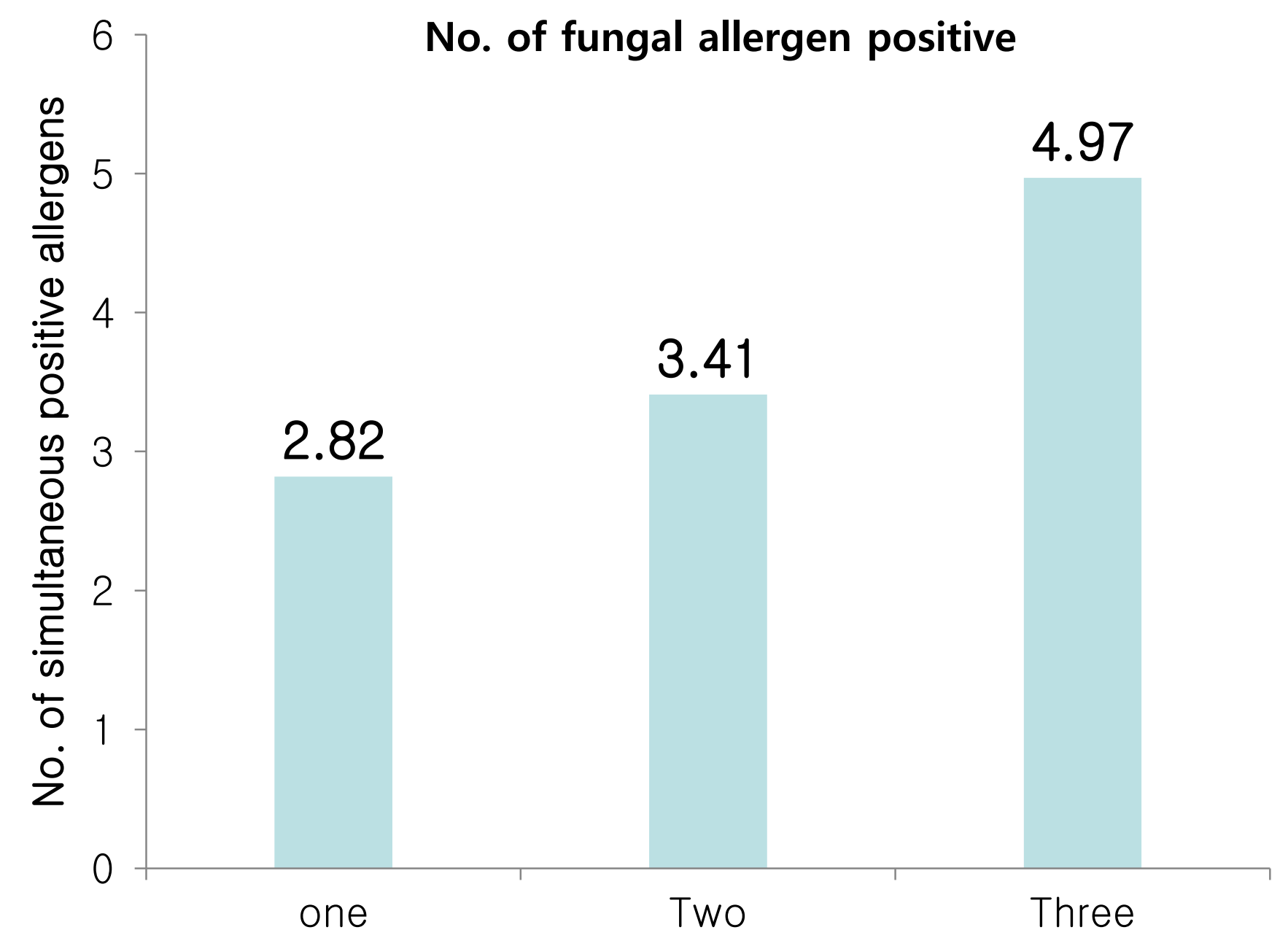

Fig 1. Simultaneous positive numbers of other allergens according to the number of fungus allergens 